\title{
Zwei wahrscheinlich letztinterglaziale Torfvorkommen im Gebiet von Stockach-Meßkirch
}

\author{
Von Kh. Göttrich, Sigmaringen und J. Werner, Freiburg \\ Mit 6 Abbildungen und 2 Tafeln
}

\begin{abstract}
$\mathrm{Zus}$ ammenfassung. Zwei Torfvorkommen in der Altmoräne des westlichen Rheingletschergebietes, beide auf sicher rißeiszeitlichen Ablagerungen und überdeckt von würmglazialen Fließerden, werden untersucht und palynologisch bearbeitet. Sie gehören mit hoher Wahrscheinlichkeit ins Riß-/Würm-Interglazial. Von keinem der beiden Vorkommen liegt ein vollständiges Pollendiagramm vor.

A bstract. Two presences of fossile peat in the western part of the Rhine Glacier Area, situated on Riss glacial deposits and under Würm glacial solifluction covers, are described and studied by geological and palynological means. With high probability they are to be placed into the Riss/Würm interglacial. In both cases the pollen diagrams are not complete.
\end{abstract}

\section{Einleitung}

Im Altmoränengebiet südwestlich Meßkirch beim „Maienberg“ (Top. Karte 1:25000 Blatt Meßkirch 8020) wurde im Jahre 1963 bei Schachtungsarbeiten für ein Hochspannungsmast-Fundament ein fossiles Torfvorkommen angefahren. Kurz darauf kam in einer Schußbohrung der PRAKLA nur wenige Kilometer südlich davon am Westrand des Moores „Waltere“ (Top. Karte 1:25000 Blatt Meßkirch 8020) ein weiterer fossiler Torf zum Vorschein. Auf Grund der geologischen Situation erschien von vornherein für beide Torfe riß/würm-interglaziales Alter wahrscheinlich. $\mathrm{Da}$ aus diesem Bereich des Rheingletschers bislang noch kein interglazialer Torf bekannt und untersucht worden war, wurden im Jahre 1964 an beiden Fundstellen Baggerschürfe angelegt und Torfproben entnommen. Die palynologische Bearbeitung hatte zunächst Herr Privatdozent Dr. G. LANG, Karlsruhe, übernommen. Da ihm jedoch aus zeitlichen Gründen die geplante Bearbeitung nicht mehr möglich war, gab er die von ihm entnommenen Proben an einen der Autoren (GöTTLICH) zur palynologischen Bearbeitung weiter. Das Ergebnis der Untersuchungen wird nunmehr vorgelegt.

\section{Geologische Situation ${ }^{1}$ )}

1. Fundort "Maienberg “

Die allgemeine Situation des Fundortes geht aus der Ubersichtskarte Abb. 1 hervor.2) Das begrabene Torfvorkommen liegt in einer flachen Senke vor einer Endmoränenkuppe (Schnitt Abb. 2). Diese ist ein Teil des morphologisch deutlichen Endmoränenzuges des W a ldbühl-Sta diums (nach der Lokalität Waldbühl bei Gallmannsweil, wo die Endmoräne in großen Kiesgruben aufgeschlossen ist). Der zum Riß-Komplex gehörende, einige Kilometer hinter der äußersten Riß-Vereisungsgrenze zurückbleibende Stand zeichnet sich durch folgende Eigenarten aus: 1. Der Endmoränenwall ist morphologisch deutlich ausgeprägt. 2. In Tagesaufschlüssen sind meist Stauchungserscheinungen feststellbar. 3. Weithin fehlen zugehörige große Übergangskegel und Schotterkörper. - Es ist sehr wahrscheinlich, daß dieses Waldbühl-Stadium des westlichen rißeiszeitlichen Rheinglet-

1) Eine ausführlichere Darstellung der rißzeitlichen Bildungen des Blattgebietes Meßkirch und seiner Umgebung wird in den Erläuterungen zur geologischen Spezialkarte 1:25 000, Blatt Meßkirch, (WERNER) erscheinen.

2) In die Karte sind weitere, noch nicht näher untersuchte überdeckte Torfvorkommen wahrscheinlich letztinterglazialen Alters eingetragen. 


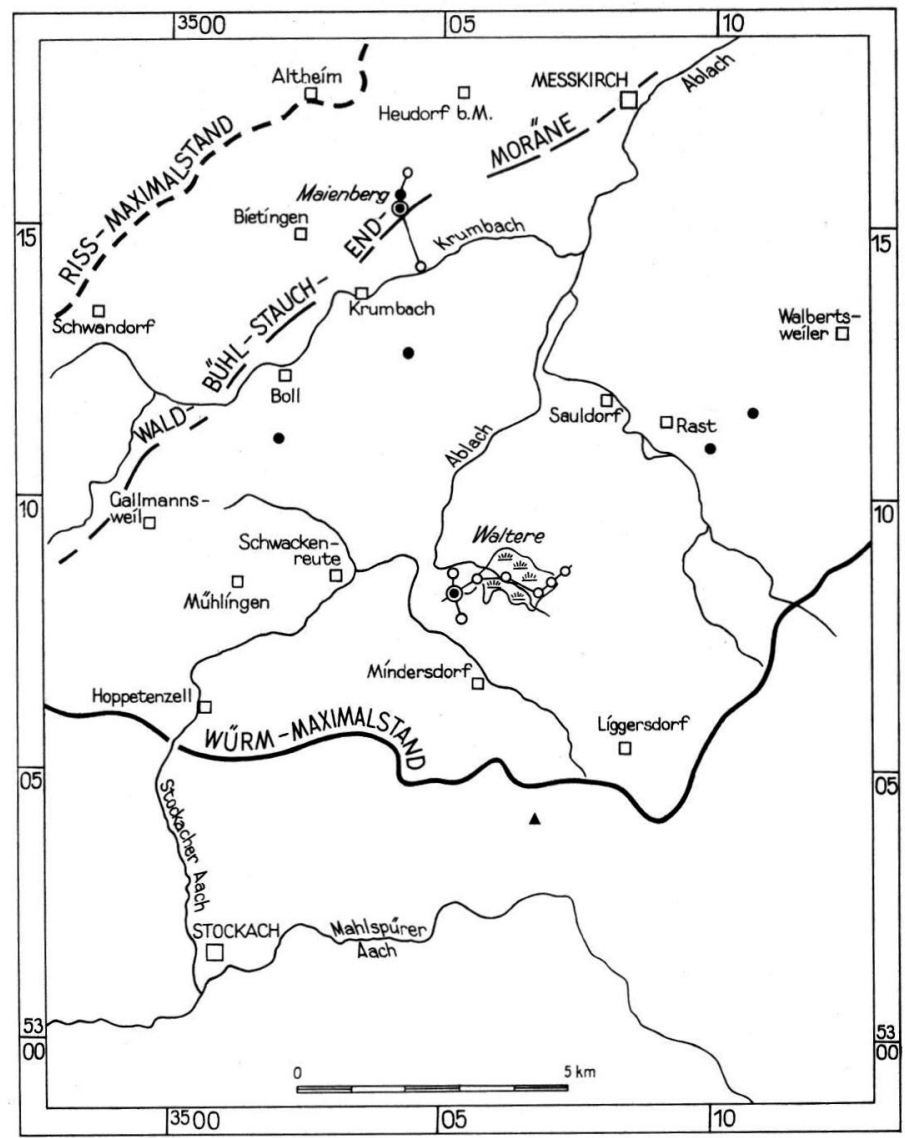

Abb. 1. Ubersichtskarte. Zeichenerklärung: $\odot=$ Aufgeschürfte, fossile Torfvorkommen Maienberg und Waltere. $\bullet=$ Sonstige in Bohrungen angefahrene fossile Torfvorkommen mit Riß-Geschiebemergel oder -Schotter im Liegenden und Fließlehm im Hangenden. $\mathrm{O}=$ Bohrungen der geologischen Schnitte Abb. 2, 4 und 5. $\boldsymbol{\Delta}=$ In einer Bohrung angefahrener fossiler Torf unter WürmGeschiebemergel-Überdeckung.

schers gleichzusetzen ist mit der das Federseebecken umrandenden Endmoräne, dem Biberacher Doppelwall (Weidenbach 1937) und den Endmoränen des Wurzacher Beckens (SCHREINER 1951).

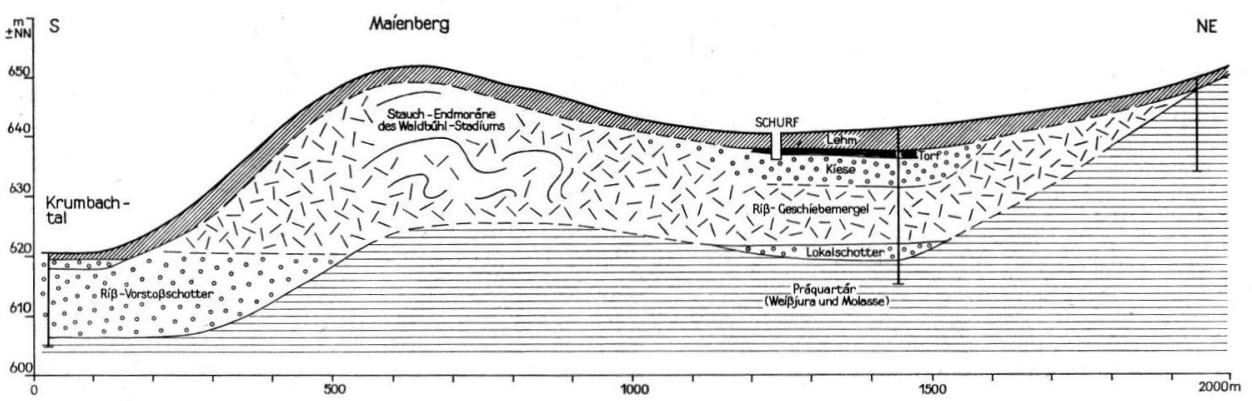

Abb. 2. Geologischer Schnitt durch das Torfvorkommen Maienberg auf Grund einiger Bohrungen und Tagesaufschlüsse (vgl. Abb. 1). 


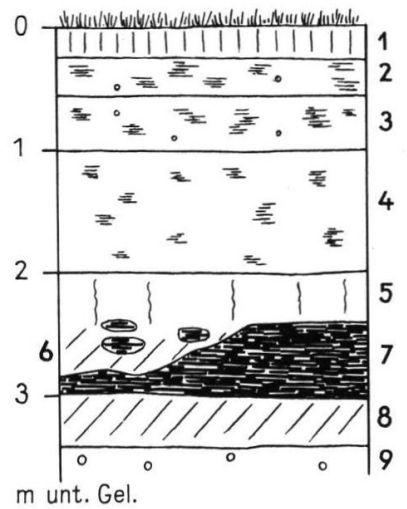

Abb. 3. Profil des Baggerschurfes beim Maienberg. $-1=$ Lehm, schluffig, fahlbraun $\left(A_{1}+g-\right.$ Horizont). 2 = Lehm, einzelne kleine Geschiebe, marmoriert ( $\mathrm{g}_{1}$-Horizont). $3=$ Lehm, einzelne kleine Geschiebe, marmoriert, Brauneisenkonkretionen ( $\mathrm{g}_{2}$-Horizont). $4=$ Lehm, einzelne kleine Geschiebe, Marmorierung nach unten abnehmend. $5=$ Lehm mit Fein- bis Mittelsand-Nestern, dicht, bläulichgrau mit Eisenhydroxydbelägen auf Kluftflächen und Wurzelröhren ( $\mathrm{G}_{0}$-Horizont). $6=$ wie 5 , jedoch von torfigen Nestern und Schlieren durchsetzt. $7=$ torfiger Lehm, schlierig durchzogen von Lehm mit geringerem Torfgehalt. Einzelne Kalkknöllchen. Wenig Holzreste, meist wohl Teile von Zweigen, eine Kiefernadel. $8=$ Lehm, schluffig-feinsandig mit Glimmer, grünlich-grau ( $\mathrm{G}_{\mathrm{r}}$-Horizont), kalkfrei. Wahrscheinlich Auelehm. $9=$ alpiner Kies, frisch, mit Anätzungsspuren.

Die flache Senke, in der das Torfvorkommen liegt, gehört zu einer den WaldbühlEndmoränenzug von Bietingen in Richtung auf Meßkirch begleitenden Talung, die als peripheres Schmelzwassertal anzusehen ist. Ihre Kiesfüllung - mit Auelehmdecke bildet das Liegende des Torfes (Abb. 3). Die Kiesfüllung ihrerseits ist in einen liegenden Geschiebemergel eingesenkt, der wohl dem Riß-Maximalstand zuzurechnen ist.

Die Oberfläche der Talung im Bereich des Torfvorkommens besitzt heute kein Längsgefälle mehr. Demnach ist das Hangende des Torfes, eine 2,5 m mächtige Überdeckung aus geschiebearmem, kalkfreiem Lehm, nicht als Auelehmbildung, sondern als Fließerde anzusehen. Diese ist ins Würmhochglazial zu stellen. Ihr Material entstammt den aus dem Geschiebemergel der Talflanken hervorgegangenen riß/würm-interglazialen Bodenbildungen (hauptsächlich wohl Parabraunerden großer Entkalkungstiefe, WERnER 1964).

Das Profil des Fundortes im einzelnen geht aus der Darstellung Abb. 3 hervor. Eine gewisse Durchbewegung des Torfes durch Kryoturbation zu Beginn der Fließerde-Überlagerung ist wahrscheinlich.

\section{Fundort"Waltere"}

Wie beim Fundort „Maienberg“ ist auch das fossile Torfvorkommen bei der „Waltere“ von Fließerde überdeckt. Die „Waltere“ (Abb. 1) ist ein mehr als 1 km² großes Hochmoor in einer durch glaziale Übertiefung entstandenen, großenteils mit Geschiebemergel verfüllten Depression. Diese hängt mit einer ebenfalls geschiebemergelverfüllten Depression der Molasse-Oberkante unter der Kiesfüllung des heutigen Ablachtales zwischen Bahnhof Schwackenreute und Sauldorf zusammen. Wahrscheinlich sind diese Depressionen als kleines und wohl relativ flaches Zungenbecken anzusehen, das beim ersten Rißgletschervorstoß (überfahrener „Meßkircher Stand“, vgl. Fußnote 1) auf Seite X) oder beim zweiten Vorstoß zum Riß-Maximalstand ausgehobelt wurde. Die Waltere liegt einige Kilometer nördlich der Äußeren Würmendmoräne und noch weit hinter dem Endmoränenzug des ebenfalls rißeiszeitlichen Waldbühl-Stadiums. 


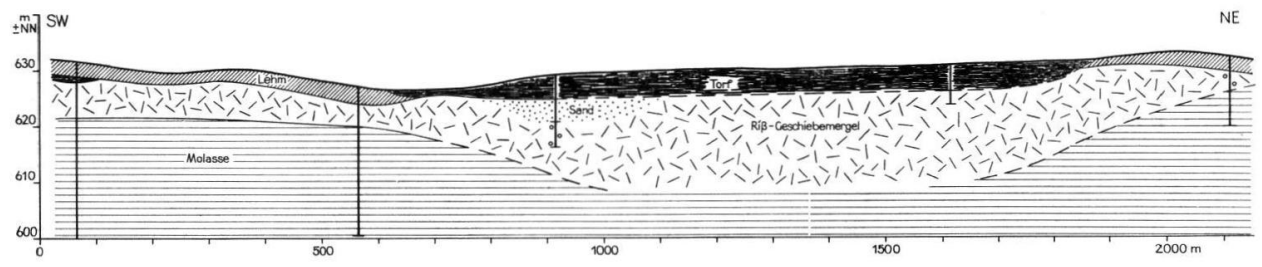

Abb. 4. Etwa westöstlich verlaufender geologischer Schnitt durch die Waltere (vgl. Abb. 1) auf Grund einiger Bohrungen.

Die Waltere greift mit ihren holozänen Torfbildungen fingerartig in eine von Südwesten her einmündende Senke hinein (Abb. 1). Nur 100 bis $200 \mathrm{~m}$ oberhalb dieses Moorausläufers wurde am Rande derselben flachen Senke das fossile Torfvorkommen entdeckt (Abb. 4 und 5). Im Liegenden des Torfes finden sich über dem Riß-Geschiebemergel ein schneckenführender Lehm und Schmitzen von Seekreide, die stellenweise kryoturbat mit dem Torf verwürgt ist (Abb. 6). Über dem Torf folgt zunächst wiederum ein schneckenführender Lehm, der von einem 2-2,5 m mächtigen kalkfreien, geschiebearmen Lehm überdeckt wird. Dieser kann nur als Fließerde gedeutet werden, die ins Würm-Hochglazial zu stellen ist.

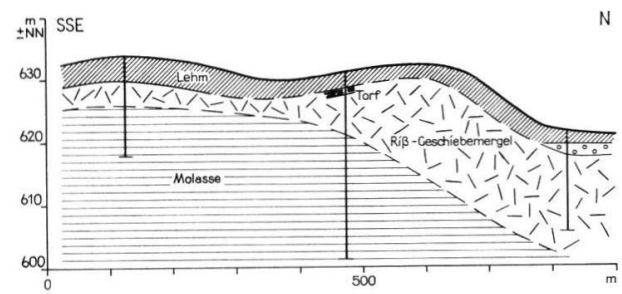

Abb. 5. Etwa südnördlich verlaufender Schnitt durch das fossile Torfvorkommen am Rande der Waltere (vgl. Abb. 1) auf Grund einiger Bohrungen.

Die Schneckenfauna vom Fundort "Waltere“

Sowohl im Torf selbst, als auch im Hangenden Lehm und in der liegenden Seekreide (Abb. 6) kommen zahlreiche Schnecken vor. Diese wurden durch Herrn Dr. Münzing, Freiburg, bestimmt, dem hierfür herzlich gedankt sei. Es treten folgende Arten auf:

Bithynia tentaculata (LinNe), Deckel

Valvata piscinalis (MülleR) ssp.

(Torf und liegende Seekreide)

Valvata piscinalis antiqua (SowerBy)

Lymnaea sp.

(Torf)

(liegende Seekreide und hangender Lehm)

(liegende Seekreide)

Es sind durchweg Süßwasserschnecken; Valvata piscinalis antiqua ist eine typische Seeform. Alle diese Arten bewohnen heute ein weites Gebiet zwischen Nordwestafrika (Bithynia tentaculata) oder Süditalien (Valvata piscinalis) bis zum hohen Norden. Die Schneckenfauna des Torfvorkommens "Waltere“ gibt somit keine Auskunft über klimatische oder stratigraphische Fragen.

\section{Absolute Altersbestimmungen}

Vom Fundort „Maienberg“ wurden drei, vom Fundort „Waltere“ zwei Torfproben durch Herrn Dr. GEYH, Hannover, nach der ${ }^{14} \mathrm{C}$-Methode datiert. Ihm sei hierfür herzlich gedankt.

Die erste in einer Torfprobe vom Fundort „Maienberg“ durchgeführte Bestimmung ergab mit $21360 \pm 420$ a ein wider Erwarten niedriges absolutes Alter. Da zu vermuten 


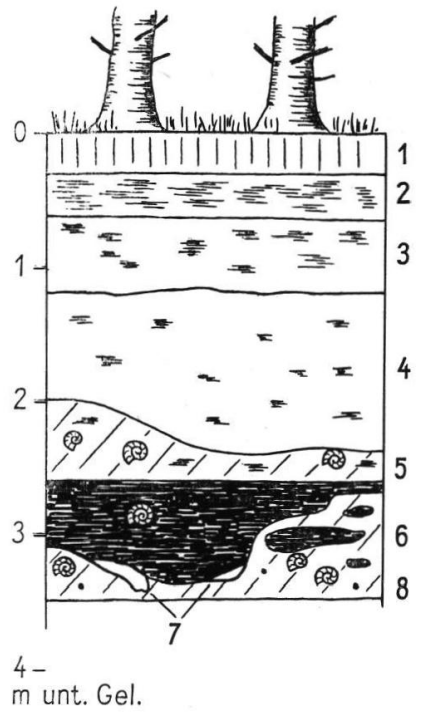

Abb. 6. Profil des Baggerschurfes bei der Waltere. $-1=$ Lehm, schluffig, braun ( $A_{1}$-Horizont). $2=$ Lehm, schluffig-feinsandig, braun und schwach marmoriert, vereinzelte Geschiebe $\left(\mathrm{B}_{t}+\mathrm{g}\right.$ Horizont). $3=$ Lehm, feinsandig, schwach marmoriert, einzelne Geschiebe. $4=$ Lehm, stark feinsandig, diffus grau und rostfleckig, einzelne Geschiebe. Bis hier kalkfrei. $5=$ Lehm, stark feinsandig-schluffig, kleinflächig bläulichgrau und rostfarben marmoriert, reichlich Schneckenschalen, einzelne Pflanzenreste. $6=$ Torf mit Holzresten, wenig Schneckenschalentrümmer. $7=$ Seekreideartiger Mergel. $8=$ Lehm, feinsandig-schluffig, dicht, blaugrau mit rostfarben umhüllten Wurzelröhren, wenig Schneckencchalen, einzelne Pflanzenreste. Darunter folgt lt. Bohrung frischer Geschiebemergel.

war, daß die rezente Durchwurzelung des Torfhorizontes eine erhebliche Fehlerquelle sei, wurden zwei weitere Proben gesiebt und ausgelesen, so daß rezente Würzelchen weitgehend ausgemerzt waren. Die hieran durchgeführten Bestimmungen ergaben für das Torfvorkommen "Maienberg“ ein Mindestalter von 41500 resp. 42900 a.

An zwei in derselben Weise vorbehandelten Torfproben vom Fundort „Waltere“ ergab sich ein $\mathrm{Mindestalter}$ von 23300 resp. 27400 a. Die Probemenge war infolge der Vorbehandlung für eine weitergehende Altersbestimmung zu gering.

$$
\text { Die Pollendiagramme (Tafel I u. II) }
$$

Darstellungs we is e

Die Analysenergebnisse wurden als Totaldiagramme aufgetragen. D.h., daß als Grundsu m me (Spalte A) alle Pollen (Baum- und Nichtbaumpollen) unter Ausschluß deren der Wasserpflanzen, herangezogen worden sind. Das jeweilige Verhältnis Baumpollen/Nichtbaumpollen ist mit einem Blick in dem ersten Diagrammteil, der nur die Baumpollen in herkömmlicher Weise enthält, sichtbar. Diese Art der Berechnung bzw. Darstellung wurde gewählt, da es sich hier um überwiegend subaquatische Sedimente handelt.

Unter den Nichtbaumpollen wurden die Arten der Frostschuttundra als schwarze Schattenrisse, die übrigen Kräuterpollen als schrägschraffierte Flächen aufgetragen.

Unter I n c e r t a sind alle die Funde zusammengefaßt, welche sich infolge zu starker Korrosion, allgemein schlechter Erhaltung, Uberdeckung durch Detritus oder aus ähnlichen Gründen nicht bestimmen ließen. Sie sind daher ein relatives Maß der Pollenerhaltung. I n d e t e r m in t a sind seltenere Einzelpollen, die mit vernünftigem Aufwand nicht mehr bestimmt werden konnten. 
Die Pollen der R a nu culace a e, wohl fast nur dem Genus Ranunculus angehörend, sind schwer und nur unsicher nach Spezies zu trennen, so daß sie zusammengefaßt wurden. Obwohl unter ihnen der Batrachium-Typus überwiegt, wurden sie sicherheitshalber nicht zu den Wasserpflanzen gestellt. Die Summe der eigentlichen Wasserpflanzenpollen (Sp. B) wäre daher, besonders beim Diagramm Maienberg, entsprechend zu erhöhen.

Spalte D enthält die Anzahl der Coenobien verschiedener Pediastren als A $1 \mathrm{~g}$ e n zusammengefaßt.

Im Diagramm Maienberg schließlich folgt darauf noch eine besondere Spalte E mit einem Sonderdiagramm, das allein die Baumpollen als Grunddiagramm zeigt.

Hingewiesen sei noch auf die Spalte Asche (jeweils links), die erkennen läßt, daß es sich fast überall um minerogene Sedimente handelt. Lediglich bei den Spektra $4-8$ von Maienberg kann aufgrund der Definition (Göttrich 1965, S. 85) von einem Anmoor gesprochen werden. Im übrigen ist eine - sehr erwünschte - paläopedologische Ansprache nicht möglich, da nur wenige $\mathrm{cm}^{3}$-große Einzelproben zur Verfügung standen.

Die Wahl der Spektraabstände $(5 \mathrm{~cm})$ war durch die Probeentnahme (G. LANG) vorgegeben.

\section{Gliederung und Deutung}

1. Die humose Schicht aus der Wa lte r e wurde schon außerhalb ihres Westrandes entnommen. Die Waltere selber ist ein holozänes, stark abgetorftes Hochmoor mit Niedermoor- und (nördlichem) Anmoorsaum.

In den basalen Spektra (bis 15) sind noch hohe Anteile von Kältezeigern vorhanden; ein (flacher) See war erst in Entwicklung begriffen. Doch auch hier sind schon, wenn auch geringe Werte, des Eichenmischwaldes als geschlossene Kurve vorhanden.

$\mathrm{Ab}$ Spektrum 15 erfolgen im Baumpollenbild bis zum Hangenden nur geringe Anderungen, wobei die Reihenfolge Pinus-Corylus-Picea-Betula-EMW - Abies fast immer eingehalten wird. Die relative Gleichförmigkeit des Baumpollendiagrammes - ineins mit der Existenz eines kleinen Sees, in dem überwiegend minerogene Ablagerungen herrscht ist als Ergebnis seiner fast ständigen Durchmischung zu deuten. Deshalb die nur geringe Gliederungsmöglichkeit.

Das BP/NBP-Verhältnis läßt auf eine nur recht lockere Bewaldung des Fundortes schließen.

Ferner ist noch festzuhalten, daß unter den Picea-Pollen keine Omorica-Typen gefunden wurden; außerdem fällt in den Spektra 1-5 eine geschlossene Kurve niedriger Frequenzen von Abies und Carpinus auf. Die Vorkommen der Wasserpflanzenpollen und der Pediastren beweisen die Entwicklung eines (kleinen) Sees mit Höhepunkt bei Spektrum 7. Das Seestadium hält - wenn auch in vermindertem Maße - bis zum Ende der untersuchten Schicht an. Die wassergefüllte Hohlform dürfte durch Zuschütten infolge Solifluktion ihr Ende gefunden haben.

Das geschlossene Vorhandensein der Lemnaceae zusammen mit der Schwarzerle deutet auf relativ eutrophe Verhältnisse im See und im Bruchwald, der ihn umgab.

2. In ähnlichem Maße wie beim vorigen ist der Baumpollenanteil des Diagrammes von

$\mathrm{M}$ a i e n be rg fast ungegliedert. Hier sind nur Pinus, Betula, Salix und Picea vorhanden, wobei die Birke nach oben von der Kiefer zurückgedrängt wird. Als tertiäre Arten an zweiter Lagerstätte wurden ein Tsuga-Fragment (Sp. 6) und zwei Tsuga-Pollen (Sp. 8) und zwei Juglandaceae (Sp. 9 und eine in Spektrum 1 gefunden).

Die Nichtbaumpollen überwiegen bei weitem, jedoch nicht so stark wie es das Diagrammbild wiedergibt. Man erkennt nämlich, daß die lokalen Cyperaceen den NBP-Anteil um etwa die Hälfte erhöhen. 
Die Umgebung der Fundstelle war nicht völlig waldfrei, jedoch sehr stark von offenen Pflanzengesellschaften durchsetzt; außerdem nahmen am Bewuchs wohl strauchförmige bzw. niederwüchsige Gattungen von Weiden, Birken, Kiefern und Fichten teil. Folgerichtig spielen hier die im Schutze eines Bruchwaldes wachsenden Farne kaum eine Rolle.

Wie im Waltere-Diagramm, doch stärker ausgeprägt, so unter anderem durch höhere Frequenzen von Pediastren, ist auch ein Seestadium mit zeitweilig etwas humusreicherer Sedimentation deutlich erkennbar.

Der flache See muß ein abruptes, katastrophenartiges Ende durch solifluktives Auffüllen und Überdecken gefunden haben. Sein üppiger Bewuchs aus Wasserhahnenfuß, Wasserlinsen und Tausendblatt ist schnell untergegangen.

3. Der Vergleich beider Diagramme läßt aus palynologischer Sicht weder eine sichere zeitliche Reihung noch die Konnektierung mit anderen Funden zu. Vermutete eemzeitliche Schichten aus Oberschwaben (GERMAN und FiLzer 1965, 1967), denen die Waltere- und Maienberg-Funde durchaus angehören können, sind kaum sicher einzuordnen, zumal meist zu fragmentarisch. Andere (Brosse, Filzer und German 1965) wären eher vergleichbar, wenn in unseren beiden Fällen nicht eine stark homogenisierende Pollenvermischung die Gliederung verwischte.

Lediglich das Pollendiagramm von der Waltere paßt - wenn man von lokalen Pollen absieht - gut mit dem der vermutlich riß/würm-interglazialen Torfe von Dürmentingen überein (GöTtLICH 1960, S. 125 ff.).

Die Radiocarbondatierung gibt hier kaum eine verwertbare Antwort; eemzeitliche Schichten müßten (Gross 1966) mindestens 75000 abp alt sein.

Die Altersangaben für die Waltere $(>24700)$ bzw. $>23300)$ sind die weniger brauchbaren, da die Schicht von feinen rezenten Wurzeln durchsetzt ist.

Für Maienberg wurden mehr als 42900 bzw. $>41500$ bestimmt. Beide Datierungen sagen nichts gegen ein riß/würminterglaziales Alter, beweisen es aber natürlich noch viel weniger. So könnte Maienberg dem Polleninhalt nach ebenso einem der vielen WürmInterstadiale angehören.

Aus diesen Gründen können für die zeitliche Zuordnung und Reihenfolge der beiden Funde nur geologische Verfahren, d. h. hier ihre stratigraphische Einordnung und ihre lateralen Beziehungen Aufschluß geben.

Herrn Priv.-Doz. Dr. H.-J. Beug, Hohenheim, sei für eine Diskussion der Diagramme an dieser Stelle herzlichst gedankt.

\section{Ergebnisse}

1. Fundort "M a i en berg “

Der fossile Torf dieses Fundortes wurde, wie das palynologische Untersuchungsergebnis zeigt, während einer kühlen Epoche sedimentiert. Ort der Torfbildung war, ebenfalls nach palynologischem Befund, ein flaches stehendes Gewässer.

Die liegenden alpinen Kiese zeigen nahe ihrer Obergrenze typische Veränderungen, wie sie durch Grundwässer unter vermoorten Oberflächen verursacht werden: Graubleichung durch Reduktion, oberflächliche Anätzung der Kalkgerölle. Zwischen Kiesen und Torfhorizont liegt ein kalkfreier Lehm von auelehmartiger Beschaffenheit. Er zeigt typische blau-graue Reduktionsfarben, wie sie im $\mathrm{G}_{\mathrm{r}}$-Horizont eines Gley-Profiles auftreten. Die Kalkfreiheit des liegenden Lehmes zeigt, daß zwischen dem Rückzug des Rißgletschers und der Sedimentation des Lehmes eine Phase der Entkalkung und Bodenbildung gelegen haben muß. Wahrscheinlich in dieser Zeit wurde der liegende Kies zersetzt und der kalkfreie Lehm in eine abgeschnittene Bachschlinge oder in einen alten Toteistümpel eingeschwemmt. Die Torfbildung in diesem ruhenden Gewässer schloß sich an, während die anorganische Sedimentation in unterschiedlichem Grade weiterging, wie der starke, 
wechselnde Lehmgehalt des Torfes zeigt. Diese Entwicklung wurde offenbar durch das Einsetzen starken periglazialen Bodenfließens abrupt abgebrochen.

Die Phase der Entkalkung und Bodenbildung ist in das lange und warme Riß/WürmInterglazial zu stellen. Die Torfbildung könnte somit der kühlen Endphase dieses Interglazials oder aber einem Altwürm-Interstadial angehören. Da für das dem Hauptwürm der äußeren Jungendmoräne vorausgehende Altwürm im Rheingletschergebiet bisher keinerlei greifbare Zeugen gefunden wurden, ist die Einstufung des Torfvorkommens in das Riß/Würm-Interglazial naheliegender. Auch das hohe Mindestalter der ${ }^{14} \mathrm{C}-\mathrm{D}$ atierung scheint für diese Zuordnung zu sprechen.

2. Fundort", Waltere"

Die Sedimentation des Torfes fällt, wie aus der palynologischen Untersuchung hervorgeht, in eine Warmzeit, anfangs jedoch noch mit Anzeichen für kühleres Klima. Sedimentations-Ort war ebenfalls ein stehendes Gewässer. Nach der Morphologie kommt hier nur ein See in Frage. Da der fossile Torf höher liegt als die Untergrenze des holozänen Torfes der Waltere (Schnitt Abb. 5) ist entweder an einen höher gelegenen, mit der Waltere selbst nicht zusammenhängenden kleineren See zu denken oder aber an das Seitenbecken eines großen Sees mit entsprechend hohem Wasserspiegel. Die Verfüllung des Sees beginnt mit einem feinsandigen, kalkreichen Lehm (Abb. 6) mit Wasserschnecken und ging über in eine seekreideähnliche Bildung, der die starke organische Sedimentation (Torfhorizont) folgte. Die Seesedimente schlie? en nach oben ab mit dem hangenden, ebenfalls wasserschneckenführenden Lehm. Erst dann folgt die Überdeckung mit kalkfreier Fließerde. - Der liegende Riß-Geschiebemergel belegt postrißglaziales Alter.

Die Torfbildung ist demnach am ehesten in das Riß/Würm-Interglazial zu stellen. Sie in ein Altwürm-Interstadial einzustufen, besteht keine Veranlassung.

\section{Literaturverzeichnis}

Brosse, P., Filzer, P. \& German, R.: Neues zur Geologie der Umgebung von Bad Wurzach. N. Jb. Geol. Paläont. Mh. 5, 255-275. 1965.

German, R. \& Filzer, P. u. a.: Ergebnisse der wissenschaftl. Kern-Bohrung Ur-Federsee 1. Oberrhein. Geol. Abh. 14, 97-139. 1965. - - Ergebnisse der wissenschaftl. Kern-Bohrung UrFedersee 2. Ebenda, 45-110. 1967.

GöttLich, Kh.: Beitr. z. Entwicklungsgeschichte der Moore in Oberschwaben. Jh. Ver. vaterl. Naturkde. Württ. 115, 93-174. 1960. - - Ergebnisse u. Ziele bodenkdl. Studien in Moor u. Anmoor. Arb. Landw. Hochschule Hohenheim 33. 1965.

Gross, H.: The So-called Göttweig Interstadial of the Würm Glaciation Current. Anthropology?, 2, 239-242, Clicago 1966.

SchreIner, A.: Diluvialgeologische Untersuchungen im Wurzacher Becken (Oberschwaben). Jber. Mitt. oberrhein. geol. Ver., N. F. 33, 78-88. 1951.

Weidenbach, F.: Bildungsweise und Stratigraphie der diluvialen Ablagerungen Oberschwabens. N. Jb. Mineral. usw. Beil.-Bd. 78 B, 66-108. 1937.

Werner, J.: Grundzüge einer regionalen Bodenkunde des südwestdeutschen Alpenvorlandes. Schriftenreihe Landesforstverw. Baden-Württ. 17, Stuttgart 1964.

Manuskr. eingeg. 8. 7. 1968.

Anschriften der Verf.: Privat-Doz. Dr. Kh. Göttlich, 748 Sigmaringen, Fürst-Friedrich-Str. 14. Landesgeologe Dr. Jörg Werner, Geol. Landesamt, 78 Freiburg i. Br., Alberstraße 5.

Den geologischen Abteilungen der Erdöl-Firmen Gewerkschaft ELWERATH und WINTERSHALL AG. sei für die freundliche Erlaubnis zur Verwendung von Schußbohrergebnissen gedankt. 